\title{
Finite element analysis of the proximal phalanx of the thumb in Hominoidea during
}

simulated stone tool use

Ana Bucchi ${ }^{1,2}$, Thomas A. Püschel ${ }^{3}$, Carlos Lorenzo ${ }^{1,2}$, Jordi Marcé-Nogué ${ }^{4,5}$

${ }^{1}$ Institut Català de Paleoecologia Humana i Evolució Social (IPHES), 4 Zona Educacional Campus Sescelades URV, Tarragona, 43007, Spain. anabuchi@gmail.com, carlos.lorenzo@urv.cat

${ }^{2}$ Area de Prehistòria, Universitat Rovira i Virgili (URV), 35 Avinguda de Catalunya, 43002 Tarragona, Spain.

${ }^{3}$ Primate Models for Behavioural Evolution Lab, Institute of Cognitive and Evolutionary Anthropology, University of Oxford, 64 Banbury Road, OX2 6PN, Oxford, United Kingdom. thomas.puschelrouliez@anthro.ox.ac.uk

${ }^{4}$ Center of Natural History, University of Hamburg, Martin Luther King Platz 3, 20146, Hamburg, Germany

${ }^{5}$ Institut Català de Paleontologia M. Crusafont, Universitat Autònoma de Barcelona, Cerdanyola del Vallès, 08193, Spain. jordi.marce.nogue@uni-hamburg.de

Corresponding author: Ana Bucchi, anabucchi@gmail.com 


\begin{abstract}
Finite element analysis was applied to analyze six individuals from different primate species (Homo sapiens, Homos sapiens neanderthalensis, Pan troglodytes, Gorilla gorilla, Pongo pygmaeus and Hylobates lar) to identify stress distribution patterns on the pollical proximal phalanx during simulated hammerstone use. We expected the stress to be better distributed in our species than in other hominids based on the idea that, unlike apes, the human hand is adapted to tool-related behaviors. Our results indicate that the human phalanx unevenly distributes stresses and is one of the most fragile of all, especially when a small hammerstone is simulated. Tool orientation relative to the phalanx did not have a substantial effect on average stress or distribution. We conclude that great apes can resist loads exerted during this activity more efficiently than humans and that there were probably other evolutionary factors acting on this bone in our species.
\end{abstract}

Keywords: Functional morphology; Hand evolution; Primates; Stress

\title{
1. Introduction
}

There is a widespread idea that the derived manual anatomy of humans is a result of selective pressures related to manipulative behaviors (e.g., Hamrick et al., 1998; Young, 2007; Key and Dunmore, 2015; Kivell et al., 2015; Skinner et al., 2015), as among primates, humans exert unique, more efficient precision and power grips (e.g., Niewoehner, 2001; Shrewsbury et al., 2003; Tocheri et al., 2003; Rolian et al., 2011; Marzke, 2013; Key and Dunmore, 2015; Bardo et al., 2017). Unlike humans, locomotion constitutes the primarily selective pressure on the hand for most primates (e.g. Tuttle, 1969; Jouffroy et al., 1991). This is not to say that non-humans primates are unable of performing tool-based activities, as they have been reported in other primates (Pruetz and Bertolani, 2007; Wynn et al., 2011; Gumert and Malaivijitnond, 2013; Visalberghi et al., 2015), and some of their tools, including stone hammers, and the behaviors involved (e.g., direct hard hammer knapping) are very similar or indistinguishable from Oldowan culture (Wynn and McGrew, 1989; Wynn et al., 2011). However, the manual 
pressures and high muscle activities experienced by the hand during travel (Susman \& Stern, 1979; Wunderlich and Jungers, 2009; Matarazzo, 2013; Samuel et al., 2018) play a more important role.

The selective pressures related to stone tool use supposedly started early in the human lineage. The intrinsic muscles of the thumb show high level forces during hard hammer percussion manufacture of Oldowan tools (Hamrick, 1998; Marzke et al., 1998). The thumb also experiences significant pressures during stone tool production (Key and Dunmore, 2015; Williams-Hatala et al., 2018). In addition, some derived morphology facilitating manipulation of stone tools, as the expanded apical tuft of the distal thumb phalanx, were already present in Orrorin (Gommery and Senut, 2006), although numerous derived conditions evolve later in a mosaic fashion until the fully derived hand of Neanderthals and modern humans (for a review, see Tocheri, 2008, and also Key and Dunmore, 2018). Changes in hominins in the shortening of the fingers relative to thumb length, which ensures the human-like precision grip capability, occurred in Australopithecus (Alba, 2003; Green and Gordon, 2008; Tocheri, 2008). Robust first metacarpals have been identified in early Homo/Paranthropus (Susman, 1988) which helps to produce stronger, efficient grips and tolerate higher joint stresses (Rolian et al., 2011; Key and Dunmore, 2015; Key and Lycett, 2018).

Even though most of the paleoanthropological literature agrees that hands of humans and nonhuman primates are adapted to different functions (i.e., manipulation vs. locomotion), recent studies have concluded that some derived traits leading towards Homo (e.g., finger proportions) are not the product of selective pressures acting directly on the hands but on other region of the skeleton (i.e., foot) (Rolian et al., 2010), which are related to terrestriality (Heldstab et al., 2016; Bardo et al., 2017), and were subsequently exapted for tool manipulation. This can explain why skillful hands were present long before the first record of lithic industry (Alba et al., 2003; Almécija et al., 2010).

How humans acquired this unique configuration of musculoskeletal traits in the hand which facilitates tool related behaviors has profound implications in our understanding of human evolution overall, considering that stone technology is a key element defining culture in our 
species (e.g., Foley and Lahr, 2003). However, testing biological causality is a hard to address and we may never be absolutely certain on the evolutionary mechanism that shaped the human hand.

Consequently, we think that new insights are needed to better assess whether the evolution of the human hand is driven by tool-related behaviors. Here we use finite element analysis (FEA) to evaluate if stress on the human pollical proximal phalanx (PP1) fits with the functional adaptation (to tool use) hypothesis for the evolution of our hand. This method makes it possible to control and repeat biomechanical scenarios under modifiable conditions (for a review see Rayfield, 2007) making it suitable for morpho-functional problems such as this. To our knowledge, no study has applied FEA to evaluate the effect of stone tool use on the hand. The stress distribution in the PP1 was analyzed in six Hominoidea taxa (Homo sapiens, Homo sapiens neanderthalensis, Pan troglodytes, Gorilla gorilla, Pongo pygmaeus and Hylobates lar) (Table 1) under loading conditions related to hard hammer percussion to describe stress patterns on PP1s during the same task. We expect to find that the human PP1s distribute stresses differently than apes, as hands in humans and apes show morphologically commitment to different functions -manipulation and locomotion (Table 2)- and the loading condition simulated here are related to stone tool use. In particular, we expect that the human phalanges distribute stresses more efficiently and can withstand the stresses related to hammerstone use better than our closest living relatives.

\section{Material and methods}

\subsection{Sample and digitalization}

A recent modern human, a Neanderthal from Krapina (Vi 202) and four extant non-human hominoids were analyzed: chimpanzee, gorilla, orangutan and gibbon. All specimens were adults with no evident pathologies (Table 1).

The PP1 from the human individual was obtained from a fresh cadaver and scanned with a micro-CT (Perkin Elmer, model Quantum Fx, Hopkinton MA, USA). The orangutan PP1 is from the Senckenberg Museum in Frankfurt (SMF 74303) and was scanned on a BIR ACTIS 
225/330 microCt scanner at the Department of Human Evolution, Max Planck Institute for Evolutionary Antrhopology (Leipzig, Germany). The remaining specimens were obtained from digital databases; the micro-CT of the Neanderthal PP1 from NESPOS (https://www.nespos.org/display/PublicNesposSpace/Human+Fossils), whereas the CT scans from the rest of the non-human sample were downloaded from the Digital Morphology Museum, KUPRI (http://dmm3.pri.kyotou.ac.jp/dmm/WebGallery/index.html). Even though KUPRI CT scans have a relative low resolution (Table 1) (they do not allow to observe bone segments in high detail), they can still provide relevant morphological information, as it has been shown that even low-resolution scans can accurately quantify shape (Slizewski et al., 2010; Gaudi et al., 2014). In fact, it has been shown that this resolution is sufficiently accurate to even identify intra-specific differences of very small specimens (Marcy et al., 2018).

In order to make models from different resolution scans comparable, trabecular bone was enclosed using the 'Convex Hull' tool in Meshlab v.2016.12 (Cignoni et al., 2008) and the same mechanical properties were defined for all the specimens. Elements were then converted to CAD models. During this last step, irregularities in the surface caused by segmentation were repaired using refinement and smoothing tools (Lautenschlager, 2016) in Geomagic Studio ${ }^{\circledR}$ (3D Systems, v. 12, Rock Hill, SC, USA).

All scans were segmented using Seg3D software (CIBC, v. 2.4.3, Utah, USA). The medullary cavity, trabeculae and compact bone were segmented on the specimens by applying a combination of case-specific thresholding values and manual painting techniques. Models of left PP1's (Table 1) were reflected to enable meaningful comparisons.

To avoid possible problems when aligning different individuals (due to inter-specific morphological differences), we selected one individual as a reference (i.e., chimpanzee) to perform a best-fit alignment to align all the models according to a common reference plane. This procedure was carried out in Geomagic Studio ${ }^{\circledR}$ (3D Systems, v. 12, Rock Hill, SC, USA) prior to FEA to align all the models so that loads could be applied in the same axis and to allow an easier interpretation of the stress results. 


\subsection{Model properties}

Structural static analysis was performed to evaluate the biomechanical behavior of the PP1s using the Finite Element Package ANSYS 17.1 in a Dell Precision ${ }^{\mathrm{TM}}$ Workstation T5500 with $48 \mathrm{~GB}$ and $5.33 \mathrm{GHz}$. Elastic, linear and homogeneous material properties were assumed for the cortical bone using the values of E (Young's modulus) 18.6 GPa and v (Poisson's ratio) 0.3, while for the trabecular bone values of E (Young's modulus) $0.75 \mathrm{GPa}$ and v (Poisson's ratio) 0.3 were assumed (Butz et al., 2012).

In this study, the focus in the comparison of the models is primarily on the von Mises stress distribution. Bone can be modeled as a brittle (Doblaré et al., 2004) or ductile (Dumont et al., 2009) material. According to Doblaré et al. (2004), the von Mises criterion is the most commonly applied and useful criterion for predicting the yield and fracture location in bone when ductile and isotropic material properties are assumed in cortical bone. The PP1 models were meshed using an adaptive hexahedral mesh and ANSYS® (Marcé-Nogué et al., 2015). The model meshes ranged between 200,000 and 320,000 elements depending on the particular specimen and loading scenario (Table 3).

\subsection{Loading scenarios and boundary conditions.}

The hands were modeled using a free-body diagram approach (Fig. 2), in a precision three-jaw chuck grip (Fig. 1a), following Marzke et al. (1998), since we obtained the applied muscle recruitment data from their study.

Forty-eight loading cases were generated. These included two hammerstone orientations relative to the PP1 and two muscle activity patterns associated with differences in hammerstone mass for the six individuals under study (Fig. 1 and 2). We also generated two different scaling scenarios for muscular data considering that for only two species (Homo sapiens and Pan troglodytes) there is enough muscular information to perform the simulations.

In the first scaling scenario we scaled the forces of the Neanderthal, chimpanzee, gorilla, orangutan and gibbon using the modern human data as a reference, whereas in the second one, 
we used the chimpanzee muscular data as a reference to scale the forces of all the other species. In each of these two settings, the loads of the remaining specimens were scaled relative to the individuals of reference to yield identical force:volume ratios (Table S1). This way differences in stress distribution can be interpreted entirely as result of shape differences (Dumont et al., 2009). These values of muscular contraction pressure were calculated according to the method developed by Marcé-Nogué et al. (2013) and rearranged for 3D models by Fortuny et al. (2015). Equation for this is $F_{A}=\left(\sqrt[3]{\frac{V_{A}}{V_{B}}}\right)^{2} F_{B}$, where $F_{A}$ is the scaled force, $F_{B}$ the reference force, and $V_{A}$ and $V_{B}$ the respective volumes for $\mathrm{CB}$.

For the human, muscle forces were calculated by means of the physiological cross-sectional area (PCSA) collected from the forearm of a fresh cadaver of a 59-year-old man. All muscles attached at the PP1 were dissected (abductor pollicis brevis [APB], extensor pollicis brevis [EPB], flexor pollicis brevis [FPB] and adductor pollicis [AP], in accordance with Sacks and Roy, 1982). The PCSA obtained from the human cadaver were the following: FPB (0.6612 $\left.\mathrm{cm}^{2}\right)$, ADP $\left(1.429 \mathrm{~cm}^{2}\right), \operatorname{EPB}\left(0.2121 \mathrm{~cm}^{2}\right)$ and APB $\left(0.2587 \mathrm{~cm}^{2}\right)$. The insertion areas of the muscles involved were defined in the model to apply the forces of muscular contraction. The angles of the muscle tendons were estimated in situ. For the remaining specimens, tendon angles were assumed to be the same as for the human (Table S1), as areas of the bone in which muscles attached are similar between them (Diogo et al., 2011a, b; Diogo et al., 2012a, Diogo et al., 2013). PCSAs for chimpanzees were obtained from Marzke et al. (1999) for the APB, FPB and AD muscles and for EPB from Kikuchi (2009). The PCSA for the chimpanzee was: FPB $\left(1.40 \mathrm{~cm}^{2}\right)$, ADP $\left(2.50 \mathrm{~cm}^{2}\right)$, EPB $\left(1.44 \mathrm{~cm}^{2}\right)$ and APB $\left(1.80 \mathrm{~cm}^{2}\right)$ (Marzke et al., 1999; Kikuchi, 2009).

The PCSA for FPB, APB and EPB muscles was then adjusted to the levels of muscle activity described in the electromyography (EMG) study of Marzke et al. (1998). These data correspond to the active (i.e., dominant) hand and was recorded at the strike, so all scenarios were simulated at that specific moment. Based on Maier and Hepp-Reymond (1995), we assumed that the activity for the APB (not monitored in the Marzke et al., 1998 study) was similar to EPB. 
Muscle activity was considered during the use of two hammerstone sizes (400 g and $780 \mathrm{~g}$, which are equivalent to $3.92 \mathrm{~N}$ and $7.65 \mathrm{~N}$ and represent the HRF). Although it would be interesting to include the loads from the core, they can significantly vary in size, as well as during the reduction sequence. More importantly, the reaction force corresponding to the core would need to be considerably higher than the hammerstone reaction force to alter the stress distribution on the PP1, which is the focus of the present study.

We simulated two tool orientations relative to the PP1: one with the long axis of the bone parallel to horizontal line (Sc 1 and 2) and the other at 45 degrees (Sc 3 and 4), as shown in Figures 1 and 2.

The hands were modeled using a free-body diagram approach. A biomechanical model (Fig. 1b) was constructed based on data about hand posture, muscles active during hammerstone use, the reaction forces from the hammerstone (HRF), and the joint reaction forces from metacarpal and distal phalanx $\left(\mathrm{JRF}_{\mathrm{mc}}\right.$ and $\mathrm{JRF}_{\mathrm{d}}$ respectively). Details of all loads involved are in Table S1.

Fig 1. Biomechanical model of hammerstone use. (b) corresponds to a zoom in palmar view of the area of interest during (a) the grip of a human individual, based on Marzke et al. (1998). (b) shows the angles of the muscular forces acting on the PP1. HRF is in 90 degrees relative to the horizontal line for scenarios 1 and 2 and in 45 degrees for scenarios 2 and 4 . This force was applied on all the palmar surface of the PP1 except in the joint areas and it is represented with a hatched rectangle. Angles of the muscle forces are shown relative to the horizontal line; $\mathrm{F}_{\mathrm{APB}}$ : Abductor Pollicis Brevis Force. $\mathrm{F}_{\mathrm{FPB}}$ : Flexor Pollicis Brevis Force. $\mathrm{F}_{\mathrm{AP}}$ : Adductor Pollicis Force. EPB direction force was applied in 16.7 degrees and is not showed here as it attached on the dorsal surface of the PP1. Grey rectangles represent the origin areas of the muscles.

Boundary conditions were defined to represent the fixed displacements that the models of PP1 experience during the loading scenarios. Once the models were solved, the joint reaction forces 
from the metacarpal $\left(J R F_{m c}\right)$ and from the distal phalanx $\left(J R F_{d}\right)$ were obtained. As boundary conditions have a great impact in the solution of the model, we intended to reproduce biological meaningful conditions for the PP1 to constrain the movements of the FEA models; the proximal part of the bone was fixed in the $\mathrm{X}$ dimension, and the distal part fixed in the $\mathrm{X}, \mathrm{Y}$ and $\mathrm{Z}$-axes (Fig. 2). All analyses were performed under these conditions (Table S1).

Figure 2. Free-body diagram of the phalanx in the different scenarios. This figure depicts the boundary conditions, areas of insertion of muscles and direction of forces. For all loading configurations, joint reaction forces resulted from the rigid boundary constraints that were fixed at the distal joint in $X, Y$ and $Z$-axes (light blue area), and at the proximal joint in the $X$-axis (dark blue area). The hammer reaction force (HRF) was applied on all the palmar surface of the bone. $3.29 \mathrm{~N}$ for the HRF was simulated for Sc 1 and 3, and $7.65 \mathrm{~N}$ for Sc 2 and 4. Phalanges are shown in palmar (right) and radial (left) views.

\subsection{Analysis of von Mises stress}

We applied the recently-proposed quasi-ideal mesh (QIM) and its percentile values (M25, M50, M75 and M95) as a basis for our analysis (Marcé-Nogué et al., 2016). The use of a QIM mesh facilitated comparison between models, which corresponds to a mesh in which all the elements have practically the same size, thus allowing the stress values obtained to be displayed as boxplots. The M95 percentile is assumed here as the peak value of stress in keeping with the idea proposed by Walmsley et al. (2013), because the maximum value cannot be properly analyzed since it corresponds to artificially inflated values (Marcé-Nogué et al., 2015). In addition, a quantitative single measurement of the relative strength of the structure under study was used to summarize the strength of the whole phalanx as the mesh-weighted arithmetic mean (MWAM) and the mesh-weighted median (MWM). These values (i.e., MWAM and MWM) are also required to estimate the percentage error of the arithmetic mean (PEofAM) and percentage error of the median (PEofM), which are statistics used to ensure that our models 
were good QIMs as described in Marcé-Nogué et al. (2016). This information can be found in the supplementary material S2 and S3.

\section{Results}

\subsection{Stress distribution.}

The distribution of von Mises stress for each phalanx and scenario is shown in Figures 3 and 4. The specimen with the highest peak stress level was the gibbon, followed by the modern human, Neanderthal, orangutan, gorilla and chimpanzee. This order was the same for all analyzed loading and scaling scenarios, except for the chimpanzee and gorilla, with the former having higher peak stresses than the gorilla in the first and second loading scenario (Homo scaled), but lower in the remaining ones (Fig. 5).

Figure 3. Von Mises stress maps for all the analyzed species under the different loading scenarios using the modern human as reference to scale the simulated muscular forces in all the other specimens. Species are ordered from higher to lower peak stress values. Phalanges are shown at the same length. Note MPa bar is set at $12 \mathrm{MPa}$.

Figure 4. Von Mises stress map for all analyzed species under different loading scenarios using the chimpanzee as reference to scale the simulated muscular forces in all the other specimens. Species are ordered from higher to lower peak stresses values. Images are not scaled. Note MPa is set at $25 \mathrm{MPa}$. A similar stress distribution to the one using the human as a reference is observed (Fig. 3).

Maximum von Mises stress values in the gibbon, modern human, Neanderthal and gorilla models were located in the center of the palmar surface of the phalanx body, decreasing towards the dorsal surface and the distal and proximal portions (Fig. 3 and 4). In these specimens, the 
lowest stress values were found in the joint areas and dorsal part, where the bone was not significantly affected by stress. Stress for the orangutan, chimpanzee and gorilla specimens were lower and more evenly distributed over the bone and, similarly to the humans and gibbon, did not affect the joint areas. In most cases, median (MWM) stress values for the modern human and Neanderthal were lower than for the chimpanzee, gorilla and orangutan (Fig. 5), although they were more concentrated than in those species (Fig. 3 and 4).

Peak and mean stress levels were considerably higher when the smaller hammerstone was simulated in all species (Fig. 5), while the effect of hammerstone orientation was less important. Overall, the effect of the hammerstone size was even greater than that related to the morphological differences between species (Fig. 5). Of all the scenarios, the one that showed the highest mean and peak values for all species was the one with the smaller hammerstone and the second bone orientation (SC 3).

Figure 5 Box-plots of von Mises stress (MPa) distribution for all species under the different scenarios, until Q95. The first row shows stress distribution of the models using the modern human as a reference to scale muscular forces in all other specimens, whereas the second one shows the results when the chimpanzee was used as a reference. Species are ordered from higher to lower peak stresses.

\section{Discussion}

Our objective was to describe the stress distribution in the PP1 of the active hand during the simulated use of hammerstones in different Hominoidea species. We varied the size of the hammerstone, the orientation of the tool relative to the hand and the muscular properties to see their effect in the stress distribution over the bone. We expected human PP1 to behave more efficiently in every scenario as we assumed its greater adaptation to tool-related behaviors. However, we found that the human phalanx was one of the most fragile bones in all cases, second only to the gibbon (Fig. 3, 4 and 5). Stress distribution in the human PP1 was uneven and the concentration at the center of the shaft indicates that it is less resistant to loads during 
forceful precision grip and is more prone to structural failure. These results indicate that nonhuman hominids (i.e., gorilla, chimpanzee and orangutan) can better withstand loads exerted during this activity as compared to humans, although other key anatomical characteristics, for instance finger proportions, facilitate this activity in the later (Napier, 1960; Rolian et al., 2011). Our results suggest that stresses in the PP1 during tool-related behaviors were not the main driving force explaining the morphology of this bone (otherwise, a different stress distribution would have been observed in the human PP1). It is possible that the selective pressures acting on the thumb during stone tool production were not as consistently high to affect the morphology of this bone. Although other studies have found that the biomechanical stresses experienced by the thumb are high during this activity (Hamrick, 1998; Marzke et al., 1998; Key and Dunmore, 2015; Williams-Hatala, 2018), there is a noticeably high variability between individuals in the observed kinematics (Rein et al., 2014), muscle activity (Marzke et al., 1998) and manual pressures (Williams-Hatala et al., 2018; Williams et al., 2012). These results raise the possibility that there were other stronger selective pressures or other evolutionary mechanisms acting on the PP1 that may not be related to stone-tool use. The argument that the evolution of the human hand was driven by selective pressures other than manipulative capabilities, has been stated before (Alba et al., 2003; Almécija et al., 2010; Rolian et al., 2010; Heldstab et al., 2016). Particularly, that the evolution of thumbs is linked to the evolution of toes (Rolian, 2009; Rolian et al., 2010) and thus locomotor functions, which comprise higher biomechanical demands than manipulation, are the primarily selective pressures shaping feet and hands in primates, including humans.

Even though the mean (MWM) and median (MWAM) stress values of humans were relatively similar to those of non-human hominoids (i.e., gorilla, chimpanzee and orangutan) (Fig. 5), the poor stress distribution observed on the human individual resulted in a more fragile PP1. The variation in the distribution of among the specimens is probably related to differences in the morphology of the PP1 (Fig. 3 and 4). Future analyses deepen the relation between PP1 morphology and stress distribution under stone tool use conditions might shed some light into this link. Previous studies provide some guidance to this problem, as anatomical variations in 
hand within and among primates has been described, such as cortices thickness (Susman, 1979; Tsegai et al., 2016), trabecular bone structure (Lazenby et al., 2011; Chirchir et al., 2015; Matarazzo; 2015; Skinner et al., 2015; Stephens et al., 2016), external dimensions (Napier, 1962; Susman, 1979; Key and Lycett, 2018) and shape of the joint surfaces (Tocheri et al., 2003, 2005; Marchi et al., 2016).

Does the force scaling of the non-human sample affect these results? While comprehensive knowledge is available about human muscles (e.g. Tuttle, 1969; Marzke et al., 1998; Diogo et al., 2012b) and in a lesser extent in chimpanzees (Marzke et al, 1999; Kikuchi, 2009), there are no analogous studies about the levels of muscular forces and activation patterns during the use of hammerstones for the other primate species studied here. To solve this problem, we scaled the muscular forces in the non-human hominins using the human and chimpanzee data as a reference, in keeping with Marcé-Nogué et al. (2013) and Fortuny et al. (2015), which allowed us to compare the behaviors of the PP1 from species that differ in size and morphology. In spite of this assumption, what makes the human (and gibbon) PP1 fragile is the stress distribution along the bone, which in this comparative analysis is not affected by the level of activation of the muscles. This becomes apparent when results from the two muscle scaling scenarios (human and chimpanzee) are compared; while stress values increase when using the chimpanzee muscles as reference, the stress distribution was very similar to the human scaling reference (Fig. 3, 4 and 5).

The validation of FEA results against experimental data to see how precisely and accurately they reflect reality was not performed. Assumptions in our study are related to muscle properties (tendon angles and forces), muscle activation patterns and muscle function for the non-human sample. Although detailed scenarios for each specimen would be ideal, these simplifications were necessary to evaluate the performance of specimens which are difficult to access or when data that is challenging to obtain (for instance, muscle activation patterns during hammer use). Therefore, simulations were used to extract general patterns between species and should be interpreted in a comparative framework. This comparative approach has been successfully 
applied in several other studies using FEA (e.g., Serrano-Fochs et al., 2015; Püschel and Sellers, 2016; Marcé-Nogué et al., 2017).

\section{Conclusions}

Non-human hominids (i.e., gorilla, chimpanzee and orangutan) can better withstand loads exerted during simulations of hard hammer percussion. Among extant Hominoidea, the human PP1s were relatively fragile at the moment of strike. Our results suggest that the forces exerted during forceful precision gripping did not act as a strong selective pressure affecting the morphology of the first pollical phalanx in humans.

\section{Acknowledgements}

We want to thank the University of Barcelona's body donation service, especially Cristina Manzanares, Juan Antonio Camara, José Luis Ramón Cayuela and Gemma Ramón Cayuela for their assistance. We are also grateful to Dr. Tracy Kivell who gave us the microCT scan data of the orangutan phalanx and to the Senckenberg Museum in Frankfurt, where this specimen is housed. We acknowledge NESPOS, and KUPRI for the CT scans of the non-human primates used in this study and to the original providers of the material: Tennoji Zoo, Fukuoka City Zoo and the Fukuchiyama City Zoo. This work was supported by the General Directorate of Research of the Spanish Ministry of Science and Technology (MICINN-FEDER) [grant number PGC2018-093925-B-C32]; the Government of Catalonia, AGAUR [grant number 2017SGR 1040]; URV [Project 2016 PFR-URV-B2-17]; the Becas Chile Program of the Chilean National Commission for Scientific and Technological Research (CONICYT); the DFG, German Research Foundation, [grant number KA 1525/9-2]; and the Government of Catalonia's CERCA program. T.A.P was funded by the Leverhulme Trust Early Career Fellowship [grant number ECF-2018-264].

\section{References}


Alba, D.M., Moyà-Solà, S., Köhler, M., 2003. Morphological affinities of the Australopithecus afarensis hand on the basis of manual proportions and relative thumb length. J. Hum. Evol. $44,225-254$.

Almécija, S., Moyà-Solà, S., Alba, D.M., 2010. Early origin for human-like precision grasping: A comparative study of pollical distal phalanges in fossil hominins. PLoS One 5, 1-10.

Bardo, A., Cornette, R., Borel, A., Pouydebat, E., 2017. Manual function and performance in humans, gorillas, and orangutans during the same tool use task. Am. J. Phys. Anthropol. $164,1-16$

Butz, K.D., Merrell, G., Nauman, E.A., 2012. A three-dimensional finite element analysis of finger joint stresses in the MCP joint while performing common tasks. HAND 7, 341-345.

Chirchir, H., Kivell, TL., Ruff, C.B., Hublin, J.J., Carlson, K.J., Zipfel, B., Richmond, B.G. 2015. Recent origin of low trabecular bone density in modern humans. PNAS 112, 366371.

CIBC, S. (2017). Seg3D. Volumetric image segmentation and visualization. Scientific Computing and Imaging Institute (SCI), Utah.

Cignoni, P., Callieri, M., Corsini, M., Dellepiane, M., Ganovelli, F., Ranzuglia, G. 2008. Meshlab: an open-source mesh processing tool. Version 1.3.2. Meshlab Visual Computing Lab, Pisa.

Diogo, R., Pastor, J.F., Ferrero, E., Barbosa, M., Potau, J., de Paz, F., Bello, G., Wood, B., 2011a. Photographic and Descriptive Musculoskeletal Atlas of Gorilla. Science Publishers, New Hampshire.

Diogo, R., Pastor, J.F., Ferrero, E.M., Burrows, A., Wood, B., Potau, J.M., de Paz, F., Aziz, M.A., Arias-Martorell, J., 2012a. Atlas of Gibbons and Siamangs Photographic and Descriptive Musculoskeletal Atlas of Gibbons and Siamangs (Hylobates). Science Publishers, New Hampshire.

Diogo, R., Richmond, B.G., Wood, B., 2012b. Evolution and homologies of primate and modern human hand and forearm muscles, with notes on thumb movements and tool use. J. Hum. Evol. 63, 64-78. 
Diogo, R., Potau, J.M., Pastor, J.F., Paz, F.J. De, Ferrero, E.M., Bello, G., Barbosa, M., Aziz, M.A., Burrows, A.M., Arias-martorell, J., Wood, B. a, 2013. Photographic and Descriptive Musculoskeltal Atlas of Chimpanzees. Science Publishers, Boca Raton.

Doblaré, M., García, J.M., Gómez, M.J.J., 2004. Modelling bone tissue fracture and healing: A review. Eng. Fract. Mech.71, 1809-1840.

Dumont, E.R., Grosse, I.R., Slater, G.J., 2009. Requirements for comparing the performance of finite element models of biological structures. J. Theor. Biol. 256, 96-103.

Foley, R., Lahr, M.M., 2003. On stony ground: Lithic technology, human evolution, and the emergence of culture. Evol. Anthropol. 12, 109-122.

Fortuny, J., Marcé-Nogué, J., Heiss, E., Sanchez, M., Gil, L., Galobart, À., 2015. 3D Bite Modeling and Feeding Mechanics of the Largest Living Amphibian, the Chinese Giant Salamander Andrias davidianus (Amphibia:Urodela). PLOS One 10, e0121885.

Gommery, D., Senut, B., 2006. The terminal thumb phalanx of Orrorin tugenensis (Upper Miocene of Kenya). Geobios 39, 372-384.

Green, D.J., Gordon, A.D. 2008. Metacarpal proportions in Australopithecus africanus. J. Hum. Evol. 54, 705-719.

Grine, F.E., Jungers, W.L., Tobias, P.V., Pearson, O.M., 1995. Fossil Homo femur from Berg Aukas, northern Namibia. Am. J. Phys. Anthropol. 97, 151-185.

Gumert, M.D., Malaivijitnond, S., 2013. Long-tailed macaques select mass of stone tools according to food type. Philos. Trans. Royal Soc. B 368, 20120413.

Hamrick, M.W., Churchill, S.E., Schmitt, D., Hylander, W.L., 1998. EMG of the human flexor pollicis longus muscle: Implications for the evolution of hominid tool use. J. Hum. Evol. $34,123-136$.

Heldstab, S.A., Kosonen, Z.K., Koski, S.E., Burkart, J.M., Van Schaik, C.P., \& Isler, K. 2016. Manipulation complexity in primates coevolved with brain size and terrestriality. Sci. Rep. 6, 24528.

Hunt, K.D. 2004. The special demands of great ape locomotion and posture. The evolution of thought: evolutionary origins of great ape intelligence, 172-189. 
Jouffroy, F.K., Godinot, M., Nakano, Y. 1991. Biometrical characteristics of primate hands. In Hands of primates. Springer, Vienna.

Key, A.J.M., Dunmore, C.J., 2015. The evolution of the hominin thumb and the influence exerted by the non-dominant hand during stone tool production. J. Hum. Evol. 78, 60-69.

Key, A.J., \& Dunmore, C. J. 2018. Manual restrictions on Palaeolithic technological behaviours. PeerJ 6, e5399.

Key, A.J., \& Lycett, S.J. 2018. Investigating interrelationships between Lower Palaeolithic stone tool effectiveness and tool user biometric variation: implications for technological and evolutionary changes. Archaeol. Anthop. Sci. 10, 989-1006.

Kikuchi, Y. 2010. Comparative analysis of muscle architecture in primate arm and forearm. J. Vet. Med. 39, 93-106.

Kivell T.L., Lemelin P., Richmond B.G., Schmitt D. (Editors), 2016. Evolution of the Primate Hand: Anatomical, Developmental, Functional and Paleontological Evidence. Springer, New York.

Lautenschlager, S., 2016. Reconstructing the past: methods and techniques for the digital restoration of fossils. Royal Soc. Open Sci. 3, 160342.

Lazenby, R.A., Skinner, M.M., Hublin, J.J., Boesch, C. 2011. Metacarpal trabecular architecture variation in the chimpanzee (Pan troglodytes): Evidence for locomotion and tool-use? Am. J. Phys. Anthropol. 144, 215-225.

Maier, M.A., Hepp-Reymond, M.C., 1995. EMG activation patterns during force production in precision grip - II. Muscular synergies in the spatial and temporal domain. Exp. Brain Res. $103,123-136$.

Marcé-Nogué, J., DeMiguel, D., Fortuny, J., de Esteban-Trivigno, S., Gil, L., 2013. Quasihomothetic transformation for comparing the mechanical performance of planar models in biological research. Palaeontol. Electron. 16, 1-15.

Marcé-Nogué, J., Fortuny, J., Gil, L., Sánchez, M., 2015. Improving mesh generation in Finite Element Analysis for functional morphology approaches. Span. J. of Palaeo. 31, 117-132. Marcé-Nogué, J., De Esteban-Trivigno, S., Escrig, C., Gil, L., 2016. Accounting for differences 
in element size and homogeneity when comparing Finite Element models: Armadillos as a case study. Palaeontol. Electron. 19, 1-22.

Marcé-Nogué, J., Püschel, T.A., Kaiser, T.M. 2017. A biomechanical approach to understand the ecomorphological relationship between primate mandibles and diet. Sci. Rep., 7, 8364.

Marchi, D., Proctor, D.J., Huston, E., Nicholas, C.L., Fischer, F., 2016. Morphological correlates of the first metacarpal proximal articular surface with manipulative capabilities in apes, humans and South African early hominins. Comptes Rendus Palevol 16, 1-15.

Marcy, A.E., Fruciano, C., Phillips, M.J., Mardon, K., Weisbecker, V. 2018. Low resolution scans can provide a sufficiently accurate, cost-and time-effective alternative to high resolution scans for 3D shape analyses. PeerJ, 6, e5032.

Marzke, M.W., Toth, N., Schick, K., Reece, S., 1998. EMG Study of Hand Muscle Recruitment During Hard Hammer Percussion Manufacture of Oldowan Tools. Am. J. Phys. Anthropol. 105, 315-332.

Marzke, M.W., Marzke, R.F., Linscheid, R.L., Smutz, P., Steinberg, B., Reece, S., \& An, K.N. 1999. Chimpanzee thumb muscle cross sections, moment arms and potential torques, and comparisons with humans. Am. J. Phys. Anthropol. 110, 163-178.

Marzke, M.W., 2013. Tool making, hand morphology and fossil hominins. Philos. Trans. Royal Soc. B 368, 20120414.

Matarazzo, S. 2013. Manual pressure distribution patterns of knuckle-walking apes. Am. J. Phys. Anthropol. 152, 44-50.

Matarazzo, S.A. 2015. Trabecular architecture of the manual elements reflects locomotor patterns in primates. PloS one, 10, e0120436.

Napier, J.R., 1960. Studies of the Hands of Living Primates. Proc. Zool. Soc. Lond. 134, 647657.

Napier, J.R., 1962. Fossil hand bones from Olduvai Gorge. Nature 196, 952-953.

Niewoehner, W.A., 2001. Behavioral inferences from the Skhul/Qafzeh early modern human hand remains. PNAS 98, 2979-2984.

Pruetz, J.D., Bertolani, P., 2007. Savanna Chimpanzees, Pan troglodytes verus, Hunt with 
Tools. Curr. Biol. 17, 412-417.

Püschel, T.A., Sellers, W.I. 2016. Standing on the shoulders of apes: analyzing the form and function of the hominoid scapula using geometric morphometrics and finite element analysis. Am. J. Phys. Anthropol. 159, 325-341.

Rayfield, E., 2007. Virtual biomechanics: Basic concepts and technical aspects of finite element analysis in vertebrate morphology. Annu. Rev. Earth, 541-76.

Rein, R., Nonaka, T., Bril, B., 2014. Movement pattern variability in stone knapping: implications for the development of percussive technologies. PLoS One 9, e113567.

Rolian, C. 2009. Integration and evolvability in primate hands and feet. Evol. Bio. 3536, 100117.

Rolian, C., Lieberman, D.E., Hallgrímsson, B., 2010. The coevolution of human hands and feet. Evolution 64, 1558-1568.

Rolian, C., Lieberman, D.E., Zermeno, J.P., 2011. Hand biomechanics during simulated stone tool use. J. Hum. Evol. 61, 26-41.

Samuel, D. S., Nauwelaerts, S., Stevens, J. M., \& Kivell, T. L. 2018. Hand pressures during arboreal locomotion in captive bonobos (Pan paniscus). J. Exp. Biol. 221, jeb170910.

Sacks, R.D., Roy, R.R., 1982. Architecture of the hind limb muscles of cats: Functional significance. J. Morphol. 173, 185-195.

Serrano-Fochs, S., De Esteban-Trivigno, S., Marcé-Nogué, J., Fortuny, J., Fariña, R.A. 2015. Finite element analysis of the Cingulata jaw: an ecomorphological approach to armadillo's diets. PloS one 10, e0120653.

Shrewsbury, M.M., Marzke, M.W., Linscheid, R.L., Reece, S.P., 2003. Comparative morphology of the pollical distal phalanx. Am. J. Phys. Anthropol. 121, 30-47.

Skinner, M.M., Stephens, N.B., Tsegai, Z.J., Foote, A.C., Nguyen, N.H., Gross, T., Pahr, D.H., Hublin, J.-J., Kivell, T.L., 2015. Human-like hand use in Australopithecus africanus. Science 347, 395-399.

Slizewski, A., Friess, M., Semal, P. 2010. Surface scanning of anthropological specimens: nominal-actual comparison with low cost laser scanner and high end fringe light projection 
surface scanning systems. Quartär 57:179-187.

Stephens, N.B., Kivell, T.L., Pahr, D.H., Hublin, J.J., Skinner, M.M. 2018. Trabecular bone patterning across the human hand. J. Hum. Evol. 123, 1-23.

Susman, R.L., 1979. Comparative and Functional Morphology of hominoid fingers. Am. J. Phys. Anthropol. 50, 215-236.

Susman, R.L., Stern, J.T., 1979. Telemetered Electromyography of Flexor Digitorum Profundus and Flexor Digitorum Superficialis in Pan troglodytes and Implications for Interpretation of the 0. H. 7 Hand. Am. J. Phys. Anthropol. 50, 565-574.

Susman, R.L. 1988. New postcranial remains from Swartkrans and their bearing on the functional morphology and behavior of Paranthropus robustus. In Evolutionary history of the "robust" Australopithecines. Aldine de Gruyter, New York.

Tocheri, M.W., Marzke, M.W., Liu, D., Bae, M., Jones, G.P., Williams, R.C., Razdan, A., 2003. Functional capabilities of modern and fossil hominid hands: three-dimensional analysis of trapezia. Am. J. Phys. Anthropol. 122, 101-112.

Tocheri, M.W., Razdan, A., Williams, R.C., Marzke, M.W. 2005. A 3D quantitative comparison of trapezium and trapezoid relative articular and nonarticular surface areas in modern humans and great apes. J. Hum. Evol. 49, 570-586.

Tocheri, M.W., Orr, C.M., Larson, S.G., Sutikna, T., Saptomo, E.W., Due, R A., Jungers, W. L. 2007. The primitive wrist of Homo floresiensis and its implications for hominin evolution. Science 317, 1743-1745.

Tocheri, M.W., Orr, C.M., Jacofsky, M.C., Marzke, M.W. 2008. The evolutionary history of the hominin hand since the last common ancestor of Pan and Homo. J. Anat., 212(4), 544-562.

Tsegai, Z.J., Stephens, N.B., Treece, G.M., Skinner, M.M., Kivell, T.L., Gee, A.H. 2017. Cortical bone mapping: an application to hand and foot bones in hominoids. Comptes Rendus Palevol, 16, 690-701.

Tuttle, R.H., 1969. Quantitative and Functional Studies on the Hands of the Anthropoidea. J. Morphol. 128, 309-363.

Visalberghi, E., Sirianni, G., Fragaszy, D., Boesch, C., 2015. Percussive tool use by Taï 
Western chimpanzees and Fazenda Boa Vista bearded capuchin monkeys: a comparison. Philos. Trans. Royal Soc. B 370, 20140351.

Walmsley, C.W., Smits, P.D., Quayle, M.R., McCurry, M.R., Richards, H.S., Oldfield, C.C., Wroe, S., Clausen, P.D., McHenry, C.R., 2013. Why the Long Face? The Mechanics of Mandibular Symphysis Proportions in Crocodiles. PLoS One 8, e53873.

Williams, E. M., Gordon, A. D., Richmond, B. G. 2012. Hand pressure distribution during Oldowan stone tool production. J. Hum. Evol. 62, 520-532.

Williams-Hatala, E. M., Hatala, K. G., Gordon, M., Key, A., Kasper, M., Kivell, T. L. 2018. The manual pressures of stone tool behaviors and their implications for the evolution of the human hand. J. Hum. Evol. 119, 14-26.

Wunderlich, R.E., Jungers, W.L. 2009. Manual digital pressures during knuckle-walking in chimpanzees (Pan troglodytes). Am. J. Phys. Anthropol. 139, 394-403.

Wynn, T., McGrew, W.C., 1989. An Ape's View of the Oldowan. Man 24, 383-398.

Wynn, T., Hernandez-Aguilar, R.A., Marchant, L.F., McGrew, W.C., 2011. “An ape's view of the oldowan" revisited. Evol. Anthropol. 20, 181-197.

Young, R.W., 2007. Evolution of the human hand: the role of throwing and. J. Anat. 202, 1-14. 